\title{
Fibrin glue increases the cell survival and the transduced gene product secretion of the ceiling culture-derived adipocytes transplanted in mice
}

\author{
Yasuyuki Aoyagi ${ }^{1,2,3}$, Masayuki Kuroda ${ }^{1,2,3,6}$, \\ Sakiyo Asada ${ }^{1,2,3}$, Hideaki Bujo, \\ Shigeaki Tanaka ${ }^{3}$, Shunichi Konno ${ }^{3}$, \\ Masami Tanio ${ }^{3}$, Itsuko Ishii ${ }^{4}$, Masayuki Aso ${ }^{3}$ \\ and Yasushi Saito ${ }^{5}$ \\ ${ }^{1}$ Center for Advanced Medicine \\ Chiba University Hospital \\ ${ }^{2}$ Department of Genome Research and Clinical Application \\ Graduate School of Medicine \\ Chiba University \\ ${ }^{3}$ CellGenTech, Inc. \\ ${ }^{4}$ Graduate School of Pharmaceutical Sciences \\ ${ }^{5}$ Chiba University \\ Chiba, Japan \\ ${ }^{6}$ Corresponding author: Tel, +81-43-222-7171, +81-43-441-4121; \\ Fax, +81-43-226-2095; E-mail, kurodam @faculty.chiba-u.jp \\ DOI 10.3858/emm.2011.43.3.021
}

Accepted 17 February 2011

Available Online 22 February 2011

Abbreviations: ccdPA, ceiling culture-derived proliferative adipocytes; LCAT, phosphatidylcholine-sterol O-acyltransferase; PPAR, peroxisome proliferator-activated receptor

\begin{abstract}
The development of clinically applicable scaffolds is important for the application of cell transplantation in various human diseases. The aims of this study are to evaluate fibrin glue in a novel protein replacement therapy using proliferative adipocytes and to develop a mouse model system to monitor the delivery of the transgene product into the blood and the fate of the transduced cells after transplantation. Proliferative adipocytes from mouse adipose tissue were transduced by a retroviral vector harboring the human lecithin-cholesterol acyltransferase (Icat) gene, and were subcutaneously transplanted into mice combined with fibrin glue. The Icat gene transduction efficiency and the subsequent secretion of the product in mouse adipocytes were enhanced using a protamine concentration of $500 \mu \mathrm{g} / \mathrm{ml}$. Adipogenesis induction did not significantly affect the Icat gene-transduced cell sur-
\end{abstract}

vival after transplantation. Immunohistochemistry showed the ectopic enzyme production to persist for 28 days in the subcutaneously transplanted genetransduced adipocytes. The increased viability of transplanted cells with fibrin glue was accompanied with the decrease in apoptotic cell death. The immunodetectable serum LCAT levels in mice implanted with the fibrin glue were comparable with those observed in mice implanted with Matrigel, indicating that the transplanted /cat gene-transduced adipocytes survived and functioned in the transplanted spaces with fibrin glue as well as with Matrigel for 28 days. Thus, this in vivo system using fibrin is expected to serve as a good model to further improve the transplanted cell/scaffold conditions for the stable and durable cell-based replacement of defective proteins in patients with LCAT deficiency.

Keywords: adipocytes; enzyme replacement therapy; fibrin tissue adhesive; phosphatidylcholine-sterol O-acyltransferase; tissue scaffolds; transplantation

\section{Introduction}

Aspirated fat is a common source of autologous tissue transplantation for the correction of tissue defects in plastic and reconstructive surgery (Billings and May, 1989; Patrick, 2000, 2001). Recent studies have shown that the preadipocytes in aspirated fat are multipotential and implicated in the source of cell-based therapies (Stashower et al., 1999; Zuk et al., 2001; Gimble et al., 2007). One such potential is the high capability for exogenous gene transduction and the secretion of transgene products (Ito et al., 2005). We have recently identified human ceiling culture-derived proliferative adipocytes (h-ccdPA) in subcutaneous adipose tissue, and proposed the application of gene-transduced $h$-ccdPA to the long-lasting replacement therapy for a variety of inherited or acquired gene-defective diseases (Asada et al., 2011; Kuroda et al., 2011).

A key factor in the protein delivery system via the autotransplantation of various types of genetransduced cells is the regulation of the survival and the secretory function of these cells at the transplanted space. We have shown that the nutri- 
tional condition of the recipient is one of the important factors for the survival and the gene expression of adipocytes in the fat graft after subcutaneous transplantation in mice (Matsumoto et al., 2002). In addition, the secretion of vascular endothelial growth factor (a bioactive molecule secreted from the vascular system) around the transplanted graft in recipients is also important for long-term cell survival (Yamaguchi et al., 2005). Particularly, recent studies have highlighted the importance of various cytokines for the regulation of cell function and the surrounding matrix conditions (Kimura et al., 2003; Cho et al., 2006; Torio-Padron et al., 2007b; Kuramochi et al., 2008; Ning et al., 2009). Together with the consideration of cytokine delivery for the transplanted cells, the development of scaffolds for transplantation contributes to the early construction of the surrounding matrix around the transplanted site. Insulin gene-transduced cells transplanted with Matrigel as a scaffold have been shown to survive as insulin-secreting adipocytes for three months after transplantation (Ito et al., 2005). It is therefore critical to set up an appropriate clinically applicable scaffold for the adipocyte transplantation into patients, which allows not only a longer survival of the implanted cells but also guarantees a longer-lasting secretion of the therapeutic gene product into the blood stream.

In this study, we have optimized the gene transduction conditions for the most effective retroviral vector-mediated gene transduction using ceiling culture-derived proliferative adipocytes from mouse adipose tissue (m-ccdPA). We established a mouse model for the transplantation with the expanded human enzyme gene-transduced m-ccdPA for the evaluation of protein delivery in the serum of the mice. Using an in vivo model, we analyzed the effect of fibrin glue (Malafaya et al., 2007; Mano et al., 2007; Neuss et al., 2008) as a clinically applicable scaffold on the efficacy of the circulating enzyme delivery.

\section{Results}

\section{Establishment of human /cat gene transduced m-ccdPA (m-ccdPA/lcat)}

We have recently established h-ccdPA, which secretes functionally active hLCAT, a key circulating enzyme for serum cholesterol esterification, and proposed a novel cell-based gene therapy by the subcutaneous transplantation of the cells for the long-lasting replacement of the protein in the patients with LCAT deficiency (Kuroda et al., 2011). In order to establish the most suitable mouse model for the evaluation of the effect of the scaffold on
A

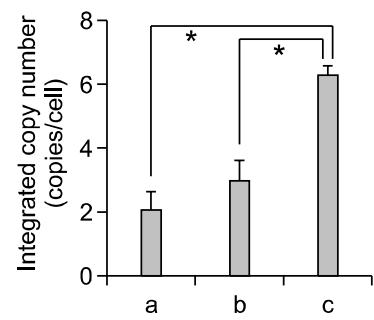

B

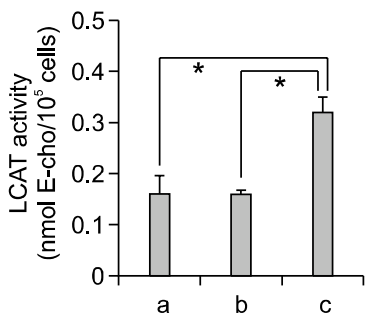

Figure 1. Enhanced gene transduction efficiency in m-ccdPA. Integrated copy numbers $(A)$ and LCAT activity in the culture medium (B) after retroviral vector-mediated human Icat gene transduction were analyzed. Single round $(b)$ and two rounds $(a, c)$ of exposure to CGT_hLCATRV in the presence of 8 (a) or $500 \mu \mathrm{g} / \mathrm{ml}(\mathrm{b}, \mathrm{c})$ of PS. Transductions with 8 $\mu \mathrm{g} / \mathrm{ml}$ (a) and $500 \mu \mathrm{g} / \mathrm{ml}$ (b, c) of PS were performed overnight and one hour, respectively. Data are presented as the mean $\pm \mathrm{SD}(n=3)$. ${ }^{*} P$ $<0.05$.

the survival and function of the transplanted adipocytes, we first prepared m-ccdPA for the Icat gene transduction as donor cells for the recipient mice. The biochemical characterization showed that the prepared m-ccdPA have morphological features and surface antigen expression patterns similar to those of h-ccdPA (unpublished data). Our preliminary experiments showed that the transduced m-ccdPA secreted a much lower amount of hLCAT than the h-ccdPA when the average copy number
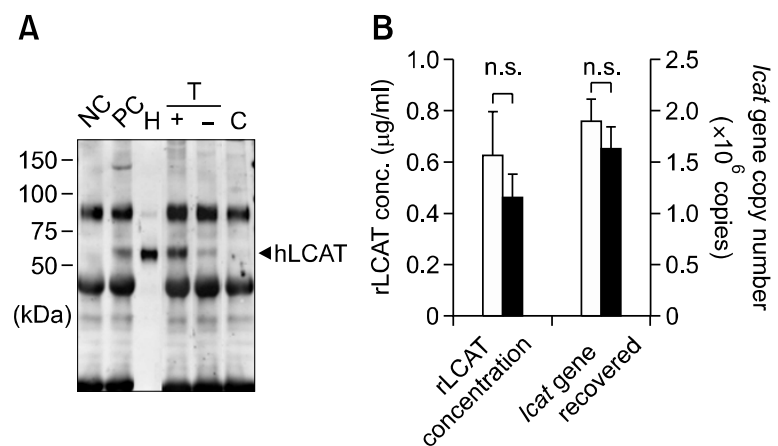

Figure 2. Detection of hLCAT and survival of human Icat gene after transplantation of lcat gene-expressing m-ccdPA. Human Icat gene-transduced mouse ccdPA $\left(5 \times 10^{6}\right.$ cells) were subcutaneously transplanted in nude mouse with fibrin glue as a scaffold. (A) Existence of hLCAT protein in mice sera was detected by IP-Western experiments. $15 \mu \mathrm{g}$ human high density lipoprotein (HDL) was loaded for quantification of signals (H). Mouse serum with (PC) or without (NC) $15 \mu \mathrm{g} \mathrm{HDL}$ were subjected to IP-Western. The gene-transduced $(\mathrm{T}) \mathrm{m}$-ccdPA were transplanted with (+) or without (-) fibrin glue. Sera (100 $\mu \mathrm{l})$ from the mice and mice transplanted with un-transduced $(C)$ m-ccdPA were subjected to IP-Western analysis. (B) Human Icat gene-transduced mouse ccdPA (5 $\times 10^{6}$ cells) were transplanted after three days of culture with (open bars) or without (closed bars) adipogenic differentiation medium. The serum concentrations of the hlcat protein were quantified by densitometric analysis (left), and the human Icat gene was quantified in excised implants (right). 
of human lcat cDNA/cell was equivalent. Two rounds of one hour exposures with CGT_hLCATRV in the presence of $500 \mu \mathrm{g} / \mathrm{ml}$ of PS significantly improved the transduction efficiency compared with two rounds of overnight exposures in the presence of $8 \mu \mathrm{g} / \mathrm{ml}$ of PS, a concentration which was originally used for the gene transduction of h-ccdPA (Figure 1A: Kuroda et al., 2011). The LCAT activity in the culture medium significantly increased in the cells with the same transduction conditions (Figure 1B).

\section{Transplantation of $\mathrm{m}$-ccdPA/lcat in nude mice}

We transplanted the above established m-ccdPA/lcat subcutaneously into nude mice to examine the effect of fibrin glue as a scaffold on the secretion of hLCAT from the surviving cells without immunoreactive conditions. Blood samples collected from the mice transplanted with or without the fibrin glue were subjected to immunoprecipitation/Western (IP-Western) procedures 7 days after transplantation (Figure 2A). hLCAT was immunologicallydetected clearly in the $\mathrm{m}$-ccdPA/lcat transplanted mice, and not in the vehicle-transplanted mice (Figure 2A). The serum from the mice transplanted with the fibrin glue showed apparently increased signal intensity in comparison to those from the mice without fibrin glue (Figure 2A), indicating that the fibrin glue is effective for the cell survival after transplantation. The signal intensity analysis suggested that the concentration of the circulating hLCAT protein is over or equivalent to those of the $15 \mu \mathrm{g}$ of human HDL, which is a major distribution site of LCAT (Fielding and Fielding, 1995).

Several reports have shown that mouse (Mizuno et al., 2008) and human (Cho et al., 2006) preadipocytes after adipogenic induction were superior in survival potential when implanted into nude mice. We therefore examined whether adipogenic differentiation affects hLCAT delivery and the survival of $\mathrm{m}$-ccdPA/lcat after implantation with fibrin glue. RT-PCR analysis showed that the PPAR 2 expression level was significantly induced in cells cultured in adipogenesis-inducing medium for three days prior to transplantation (data not shown). The serum hLCAT concentration was not significantly different between mice transplanted with differentiation-induced cells and those transplanted with uninduced cells (Figure 2B). The lcat gene recovery analysis suggested that the adipogenesis-inducing pretreatment did not affect the cell survival rate (Figure 2B). These results indicate that the transplanted m-ccdPA/lcat implanted with fibrin glue survive at least 14 days after transplantation in immunosuppressive conditions.
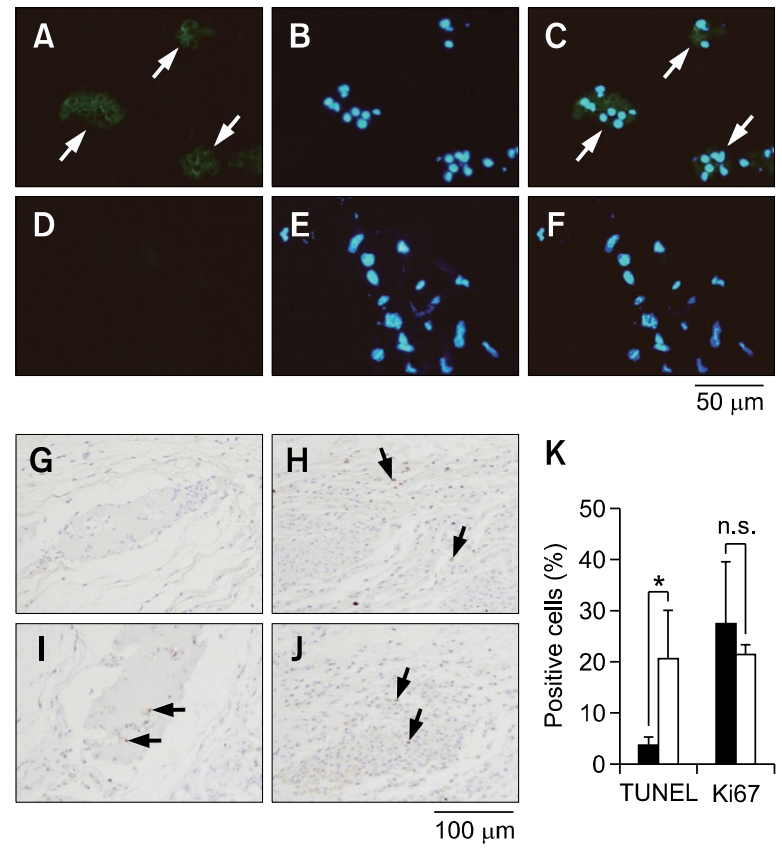

$\mathrm{K}$

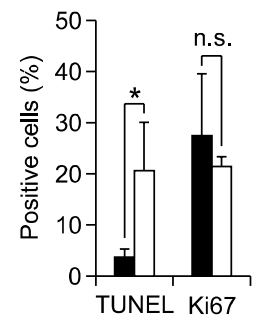

Figure 3. Immunohistochemical analysis of transplanted Icat gene-expressing $m-c c d P A$. Sections of implants from the cells with transduced $(A, B$, and $C)$ or un-transduced $(D, E$, and $F)$ by retroviral vector at day 28 were prepared and LCAT-immunostaining was performed. Implants were taken upon observation of PKH26 fluorescence. Immunohistochemical staining of hLCAT in fixed implants was done using rabbit anti-hLCAT monoclonal antibody as a primary antibody. Alexa Fluor 488 goat anti-rabbit lgG was used as a secondary antibody. The slides were counterstained with DAPI. Photographs of hLCAT staining (A and D), $D A P I$ staining $(B$ and $E)$, and merged images $(C$ and $F)$ were shown. TUNEL ( $G$ and $H$ ) and Ki67 (I and J) staining of explants on 15 days after transplantation with $(G$ and $\mathrm{I})$ or without $(\mathrm{H}$ and $\mathrm{J}$ ) fibrin glue were performed. Cells with positive signal were counted in four independent areas $(\mathrm{K})$. ${ }^{*} P<0.05$.

\section{Effect of fibrin glue on hLCAT delivery in m-ccdPA/lcat-transplanted B6 mice}

We and others have already shown that exogenous gene-transduced adipocytes survive more than 28 days when subcutaneously transplanted with Matrigel, which is used as an experimental scaffold in many studies (Kitagawa and Kawaguchi, 1999; Ito et al., 2005; Piasecki et al., 2008). In order to consider the possibility of fibrin glue as a clinical scaffold, we analyzed the effect of fibrin glue on hLCAT delivery in comparison to Matrigel in B6 mice. The m-ccdPA/lcat was subcutaneously transplanted into B6 mice with fibrin glue. hLCAT immunostaining revealed that the $\mathrm{m}$-ccdPA survive 14 days after transplantation and express hLCAT protein in B6 mice (Figures 3A-3F). The hLCAT expression was still detectable in the transplanted m-ccdPA 28 days after transplantation (data not shown). The TUNEL staining of transplanted sections excised on 15 days after transplantation showed that the 
A

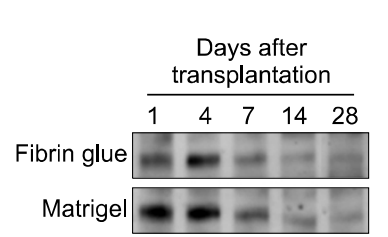

B

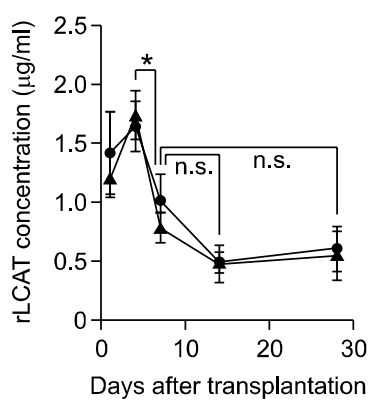

Figure 4. Effect of fibrin glue on hLCAT protein delivery. Human Icat gene-transduced m-ccdPA were subcutaneously transplanted in C57BL/6J mice using fibrin glue or Matrigel as scaffolds. Representative data of the experiments were shown (A), in which hLCAT delivery was monitored in a single mouse. Concentrations of hLCAT protein in cell-transplanted mice sera with Matrigel (closed circle) or fibrin glue (closed triangle) were quantified by densitometric analysis after IcP-Western experiments (B). ${ }^{*} P<0.05$.

apoptotic cell death in the cells with fibrin glue was significantly decreased in comparison to those without fibrin glue (Figures 3G, 3H and $3 \mathrm{~K}$ ). On the other hand, there was no significant difference in Ki67 staining between the cells with and without fibrin glue (Figures $3 \mathrm{I}, 3 \mathrm{~J}$ and $3 \mathrm{~K}$ ). The IP-Western analysis showed that the hLCAT protein was detected at least up to 28 days after transplantation in the serum of mice. The collected mouse sera were analyzed for hLCAT protein by IPWestern blotting and hLCAT protein was detected up to 28 days after transplantation (Figure 4A). Densitometric analysis (Figure 4B) showed that from day 4 to day 7 , the density became significantly decreased, and from day 7 the concentration of hLCAT protein became relatively constant. The hLCAT levels in the serum from m-ccdPA/lcat transplanted with fibrin glue were comparable with those from $\mathrm{m}$-ccdPA/lcat transplanted with the Matrigel reagent. These results showed that fibrin glue, a common clinically available material, worked as a scaffold for the in vivo delivery of the hLCAT protein.

\section{Discussion}

For the development of long-lasting protein replacement therapy by gene-transduced ccdPA, the use of a clinically applicable scaffold is one plausible approach for the improvement of survival and/or secretion function of transplanted cells in patients. Various types of materials have been proposed (Malafaya et al., 2007; Mano et al., 2007; Neuss et al., 2008) as scaffolds for cell transplantation. In this study, we have chosen fibrin glue because it is already commonly used in clinics and an easy-touse kit is commercially available. In order to evaluate the effect of fibrin glue as a scaffold in the survival and function of transplanted adipocytes, we established an autologous mouse model system using $\mathrm{m}$-ccdPA/lcat. The results using the mice showed that fibrin glue supported human enzyme delivery from the transplanted m-ccdPA/ Icat at a level equivalent to Matrigel, which is known as an efficient scaffold in experimental models. Thus, fibrin glue could be a candidate as a scaffold in the clinical transplantation of h-ccdPA/ Icat in LCAT-deficient patients to prevent the development of renal insufficiency and/or corneal opacity.

Preliminary experiments showed that hLCAT protein was secreted by $\mathrm{m}$-ccdPA/lcat in vitro, however, hLCAT was barely detectable in the transplanted mouse serum probably because of the lower capability of secretion. The integrated copy number and the LCAT activity in the culture medium could be elevated approximately three fold with the conditions suitable for m-ccdPA (Figure 1). As a result, IP-Western analysis was sensitive enough for the quantification of the serum hLCAT protein in the mice, and the analysis indicated that the delivered protein is equivalent to that of $15 \mu \mathrm{g}$ of HDL (Figure 2A). These optimizations enabled us to establish an in vivo mouse model to monitor the effect of fibrin glue as a scaffold for the transplanted m-ccdPA. Adipogenic differentiation did not significantly affect the hLCAT delivery and the cell survival in this model using fibrin glue as a scaffold with m-ccdPA (Figure 2B). In this context, our results may suggest that the transplanted cells with fibrin glue were differentiated into adipocytes without adipogenic pretreatment (Cho et al., 2006; Torio-Padron et al., 2007a). The immunohistochemical observation did not clearly show that ccdPA would undergo adipogenic differentiation after transplantation, but the transplanted ccdPA were clearly identified as hLCAT-delivery cells in the transplanted sites of the recipient mice (Figures 3A-3F). Immunohistochemical analysis of transplanted sections suggested that action of fibrin glue was prevention of apoptotic cell death rather than proliferation stimulation of the transplanted cells after transplantation (Figures 3G-3K), and thus, caused the increase in the hLCAT-delivery into circulation after transplantation in mice. The analysis of the $m$-ccdPA/lcat with fibrin glue revealed that the serum hLCAT concentration decreased to one half in a week, and became relatively stable at 7-14 days after transplantation (Figure 4). We could therefore discern that the hLCAT-positive cells survived and functioned for at least one month 
using the m-ccdPA/lcat transplanted mouse model.

The current study showed that the implanted cells successfully supplied a therapeutic level of hLCAT into the serum, and suggested the feasibility of ccdPA-mediated gene therapy using the ccdPA. However, there are several remaining issues to be resolved before the clinical application of this therapy if we anticipate extending this cell implantation technique to various diseases other than LCAT deficiency. First, the survival period of ccdPA needs to be assessed after transplantation into the recipient. The previous model using insulin-secreting adipocytes showed that the blood glucose-reducing activity was stably observed for two months (Ito et al., 2005). The stability of the ccdPA needs to be evaluated for longer periods using the mice established in this study. Second, the protein delivery by the transplanted ccdPA into the serum is unstable at the initial phase to 7 days after subcutaneous transplantation, although the delivery became constant after the 7-day phase up to a month. The characterization of the transplanted ccdPA including the interaction between the differentiation and secretion functions is in progress using this model. Before obtaining the knowledge of the multi-phase cell conditions in the recipients, the application of this cell therapy would be restricted to the enzyme deficiency in recipients without the overdose toxicity in the enzyme-mediated metabolism. In order to resolve the above remaining problems for wide clinical applications, the established autologous cell transplantation model enables us to evaluate the effects of the environmental conditions of the transplanted ccdPA on the survival and/or function of cells in detail, which is critical for successful cell-based gene therapies in humans.

\section{Methods}

\section{Cell culture}

Dulbecco's modified Eagle's medium/F12-HAM (SigmaAldrich, St. Louis, MO) supplemented with $20 \%$ fetal bovine serum (FBS, SAFC Biosciences, Lenexa, KS) and $40 \mu \mathrm{g} / \mathrm{ml}$ gentamicin (GENTACIN, Schering-Plough Co., Kenilworth, NJ) was used as culture media except for the adipogenic induction in which PGM-2 Bullet kit (Lonza, Basel, Switzerland) was used. The m-ccdPA were prepared from 7-8 weeks male C57BL/6J mice as described (Kuroda et al., 2011).

\section{Optimization of gene transduction}

Human Icat gene-expressing amphotropic retrovirus vector, CGT_hLCATRV (Kuroda et al., 2011), was used for gene transduction at the concentration of $2.0 \times 10^{9}$ RNA copies/ $\mathrm{ml}$. Based on the report of Landazuri et al. (2007), we examined $100-500 \mu \mathrm{g} / \mathrm{ml}$ of protamine sulfate (PS, NovoProtamine Sulfate, $100 \mathrm{mg}$ for I.V. Injection, Mochida Pharm. Co. Tokyo, Japan) to enhance transduction efficiency in comparison to $8 \mu \mathrm{g} / \mathrm{ml}$. Gene transduction was performed at $37^{\circ} \mathrm{C}$ in the presence of $20 \%$ FBS and PS. Subsequently, LCAT activities secreted in culture medium were measured to examine the effect of transduction conditions using artificial liposome substrate as described (Kuroda et al., 2011).

\section{Real-time PCR and RT-PCR}

Genomic DNA extractions from cultured cells and mice transplants, and quantification of transduced human lcat gene were performed as described (Kuroda et al., 2011). Total RNA was prepared by RNeasy Plus Mini kit (QIAGEN). Single-stranded cDNA was synthesized with ReverTra Ace- $\alpha^{-{ }^{T M}}$ kit (TOYOBO, Osaka, Japan). PPAR $\gamma 2$ expression was examined by RT-PCR using primers as follows; PPAR $\gamma 2-F$ (5'-GGTGAAACTCTGGGAGATTC-3') and PPAR $\gamma 2-R$ (5'-CAACCATTGGGTCAGCTCTTG-3'). The amplification was performed with TITANIUM Taq DNA polymerase (TaKaRa Bio Inc.) under the following condition: $94^{\circ} \mathrm{C}$ for $5 \mathrm{~min} / 94^{\circ} \mathrm{C}$ for $30 \mathrm{~s}, 58^{\circ} \mathrm{C}$ for $30 \mathrm{~s}$, and $72^{\circ} \mathrm{C}$ for $90 \mathrm{~s}$ ( 28 cycles) $/ 72^{\circ} \mathrm{C}$ for $7 \mathrm{~min}$. The amplified products were subjected to $2 \%$ agarose gel electrophoresis and visualized with staining with GelStar ${ }^{\circledR}$ Nucleic Acid Stain reagent.

\section{Detection of LCAT protein}

Mice sera were diluted up to $500 \mu \mathrm{l}$ with ice-cold phosphate buffered saline containing $0.2 \%$ Nonidet P-40 (PBSNP40) and incubated with $2.5 \mu \mathrm{l}$ of anti-LCAT rabbit monoclonal antibody (EPITOMICS, Burlingame, CA) overnight at $4^{\circ} \mathrm{C}$ with rotation. Twenty microliters of TrueBlot ${ }^{\mathrm{TM}}$ anti-Rabbit Ig IP Beads (eBioscience, San Diego, CA) was added and incubated with rotation for $2 \mathrm{~h}$ at $4^{\circ} \mathrm{C}$. Beads/proteins complex was washed with PBS-NP40, and treated by boiling in $10 \mu \mathrm{l}$ of $2 \times$ Laemmli's sample buffer. Samples and standards (recombinant human LCAT (Roar Biomedical, Inc., New York, NY) or human plasma HDL (Calbiochem, Merck, Darmstadt, Germany)) were subjected to western blotting using anti-LCAT rabbit polyclonal antibody (Novus Biologicals, Littleton, $\mathrm{CO}$ ) and TrueBlot anti-Rabbit IgG HRP (1:5000) (eBioscience) as primary and secondary antibody, respectively. The signals were detected by SuperSignal ${ }^{\mathbb{R}}$ West Femto Maximum Sensitivity Substrate (Thermo Fisher Scientific Inc.) with LAS1000 apparatus (FUJI film, Tokyo, Japan). Preliminary experiments demonstrated that the efficiency of recovery of input human LCAT (hLCAT) as HDL into mice serum was 101.0 $\pm 9.5 \%$.

\section{In vivo experiment}

Animal experiments were carried out according to the Guidelines for Animal Research of Chiba University or 
ORIENTAL YEAST Co., Ltd.. Male nude and C57BL/6J mice (Charles River Japan) were used as recipients. The cells were stained using $\mathrm{PKH} 26$ Red Fluorescent Cell Linker kit for General Cell Membrane Labeling (SigmaAldrich) to identify the transplanted cells in vivo.

Bolheal (The Chemo-Sero-Therapeutic Research Institute, Kumamoto, Japan) was used as clinically available fibrin glue. Fibrinogen solution and thrombin solution were diluted four and two times using DMEM-HAM/F12 (Sigma) respectively before use. Cells were suspended at $1 \times 10$ cells $/ \mathrm{ml}$ by diluted thrombin solution, and injected subcutaneously into the mouse with same volume of diluted fibrinogen solution using injection apparatus included in Bolheal kit. We also transplanted the cells suspended in Matrigel (BD Biosciences, Bedford, MA) at $5 \times 10^{6}$ cells/ $\mathrm{ml}$. In both cases, $5 \times 10^{6}$ cells were transplanted.

All mice were allowed free access to regular chow and water. Three animals were sacrificed to take serum samples at day 1, 14, 28. In C57BL/6J mice experiments, blood samples were taken from tail without sacrifice to monitor the hLCAT delivery in same animal at day 1, 4, 7, 14 and 28. Transplanted region was taken under fluorescent microscopic observation by SZX16 reflected fluorescence system (OLYMPUS corp. Tokyo, Japan).

\section{Histological staining}

The explanted tissues were fixed in $4 \%$ paraformaldehyde following replaced $30 \%$ gum-saccarose and embedded in Tissue-Tek O.C.T. Compound (Sakura Finetechnical Co., Ltd, Tokyo, Japan). Immunohistochemical staining was performed using anti-LCAT rabbit monoclonal antibody (250:1; EPITOMICS) and Alexa Fluor 488 goat anti-rabbit IgG (1000:1; Invitrogen) as primary and secondary antibody, respectively. The slides were counterstained with DAPI using VECTASHIELD Mounting Medium with DAPI (Vector Laboratories, Inc., Burlingame, CA). TUNEL staining of the explanted tissues were performed using In situ Apoptosis Detection Kit (TaKaRa Bio Inc., Shiga, Japan). Ki67 imuunostaining was performed using anti-mouse Ki67 Rabbit polyclonal antibody (Abcam plc., Cambridge, UK), followed by biotin-conjugated anti-Rabbit Ig/HRP-conjugated streptavidin reaction. Signals were visualized by HRP reaction with $D A B$ and the slides were counterstained with hematoxylin for TUNEL and Ki67 staining.

\section{Statistical analysis}

Data are presented as means \pm S.D. Statistical comparison were made by Student's $t$-test or by ANOVA followed by the post hoc Tukey test to compare using SPSS software. In all cases, $P$ values of less than 0.05 were considered as significant.

\section{Acknowledgements}

This study was supported in part by Health and Labour Sciences Research Grants for Translational Research, Japan (H. B.).

\section{References}

Asada S, Kuroda M, Aoyagi Y, Bujo H, Tanaka S, Konno S, Tanio M, Ishii I, Aso M, Saito Y. Disturbed apolipoprotein A-I-containing lipoproteins in fish-eye disease are improved by the lecithin:cholesterol acyltransferase produced by gene-transduced adipocytes in vitro. Mol Genet Metab 2011;102:229-31

Billings E Jr, May JW Jr. Historical review and present status of free fat graft autotransplantation in plastic and reconstructive surgery. Plast Reconstr Surg 1989;83:368-81

Cho SW, Kim I, Kim SH, Rhie JW, Choi CY, Kim BS. Enhancement of adipose tissue formation by implantation of adipogenic-differentiated preadipocytes. Biochem Biophys Res Commun 2006;345:588-94

Fielding CJ, Fielding PE. Molecular physiology of reverse cholesterol transport. J Lipid Res 1995;36:211-28

Gimble JM, Katz AJ, Bunnell BA. Adipose-derived stem cells for regenerative medicine. Circ Res 2007;100:1249-60

Ito M, Bujo H, Takahashi K, Arai T, Tanaka I, Saito Y. Implantation of primary cultured adipocytes that secrete insulin modifies blood glucose levels in diabetic mice. Diabetologia 2005;48:1614-20

Kimura Y, Ozeki M, Inamoto T, Tabata Y. Adipose tissue engineering based on human preadipocytes combined with gelatin microspheres containing basic fibroblast growth factor. Biomaterials 2003;24:2513-21

Kitagawa $\mathrm{Y}$, Kawaguchi $\mathrm{N}$. De novo adipogenesis for reconstructive surgery. Cytotechnology 1999;31:29-35

Kuramochi D, Unoki H, Bujo H, Kubota Y, Jiang M, Rikihisa $\mathrm{N}$, Udagawa A, Yoshimoto S, Ichinose M, Saito Y. Matrix metalloproteinase 2 improves the transplanted adipocyte survival in mice. Eur J Clin Invest 2008;38:752-9

Kuroda M, Aoyagi Y, Asada S, Bujo H, Tanaka S, Konno S, Tanio M, Ishii I, Machida K, Matsumoto F, Satoh K, Aso M, Saito $Y$. Ceiling culture-derived proliferative adipocytes are a possible delivery vehicle for enzyme replacement therapy in lecithin:cholesterol acyltransferase deficiency. Open Gene Ther J 2011; In press

Landazuri N, Krishna D, Gupta M, Le Doux JM. Retroviruspolymer complexes: study of the factors affecting the dose response of transduction. Biotechnol Prog 2007;23:480-7

Malafaya PB, Silva GA, Reis RL. Natural-origin polymers as carriers and scaffolds for biomolecules and cell delivery in tissue engineering applications. Adv Drug Deliv Rev 2007;59:207-33

Mano JF, Silva GA, Azevedo HS, Malafaya PB, Sousa RA, Silva SS, Boesel LF, Oliveira JM, Santos TC, Marques AP, Neves NM, Reis RL. Natural origin biodegradable systems in tissue engineering and regenerative medicine: present status and some moving trends. J R Soc Interface 2007; 4:999-1030

Matsumoto F, Bujo H, Kuramochi D, Saito K, Shibasaki M, Takahashi K, Yoshimoto S, Ichinose M, Saito Y. Effects of nutrition on the cell survival and gene expression of transplanted fat tissues in mice. Biochem Biophys Res 


\section{Commun 2002;295:630-5}

Mizuno $\mathrm{H}$, Itoi $\mathrm{Y}$, Kawahara S, Ogawa R, Akaishi S, Hyakusoku $\mathrm{H}$. In vivo adipose tissue regeneration by adipose-derived stromal cells isolated from GFP transgenic mice. Cells Tissues Organs 2008;187:177-85

Neuss S, Apel C, Buttler P, Denecke B, Dhanasingh A, Ding X, Grafahrend D, Groger A, Hemmrich K, Herr A, JahnenDechent W, Mastitskaya S, Perez-Bouza A, Rosewick S, Salber J, Wöltje M, Zenke M. Assessment of stem cell/ biomaterial combinations for stem cell-based tissue engineering. Biomaterials 2008;29:302-13

Ning H, Liu G, Lin G, Yang R, Lue TF, Lin CS. Fibroblast growth factor 2 promotes endothelial differentiation of adipose tissue-derived stem cells. J Sex Med 2009;6:967-79

Patrick CW Jr. Adipose tissue engineering: the future of breast and soft tissue reconstruction following tumor resection. Semin Surg Oncol 2000;19:302-11

Patrick CW Jr. Tissue engineering strategies for adipose tissue repair. Anat $\operatorname{Rec} 2001 ; 263: 361-6$

Piasecki JH, Moreno K, Gutowski KA. Beyond the cells: scaffold matrix character affects the in vivo performance of purified adipocyte fat grafts. Aesthet Surg J 2008;28:306-12
Stashower M, Smith K, Williams J, Skelton H. Stromal progenitor cells present within liposuction and reduction abdominoplasty fat for autologous transfer to aged skin. Dermatol Surg 1999;25:945-9

Torio-Padron N, Baerlecken N, Momeni A, Stark GB, Borges $\mathrm{J}$. Engineering of adipose tissue by injection of human preadipocytes in fibrin. Aesthetic Plast Surg 2007a;31: 285-93

Torio-Padron N, Borges J, Momeni A, Mueller MC, Tegtmeier FT, Stark GB. Implantation of VEGF transfected preadipocytes improves vascularization of fibrin implants on the cylinder chorioallantoic membrane (CAM) model. Minim Invasive Ther Allied Technol 2007b;16:155-62

Yamaguchi M, Matsumoto F, Bujo H, Shibasaki M, Takahashi K, Yoshimoto S, Ichinose M, Saito Y. Revascularization determines volume retention and gene expression by fat grafts in mice. Exp Biol Med (Maywood) 2005;230:742-8

Zuk PA, Zhu M, Mizuno H, Huang J, Futrell JW, Katz AJ, Benhaim P, Lorenz HP, Hedrick MH. Multilineage cells from human adipose tissue: implications for cell-based therapies. Tissue Eng 2001;7:211-28 